\title{
Motional resonances in a molecular synchrotron
}

\author{
Cynthia E. Heiner, ${ }^{1}$ Gerard Meijer, ${ }^{1}$ and Hendrick L. Bethlem ${ }^{1,2}$ \\ ${ }^{1}$ Fritz-Haber-Institut der Max-Planck-Gesellschaft, Faradayweg 4-6, 14195 Berlin, Germany \\ ${ }^{2}$ Laser Centre Vrije Universiteit, De Boelelaan 1081, NL-1081HV Amsterdam, The Netherlands
}

(Received 23 May 2008; published 23 September 2008)

\begin{abstract}
A molecular synchrotron offers unique possibilities to study molecular collisions at low energies. For such a study, it is imperative that the velocity of the stored molecules can be tuned over a wide range. Bunching elements, used to provide the necessary longitudinal focusing forces in a synchrotron, introduce instabilities for certain velocities of the stored molecules. Here we present an experimental study of these motional resonances and show how they can be avoided. A packet of $\mathrm{ND}_{3}$ molecules is stably confined in the synchrotron as its injection velocity is continuously scanned from $60 \mathrm{~m} / \mathrm{s}\left(E_{\mathrm{kin}}=3.0 \mathrm{~cm}^{-1}\right)$ to $87.5 \mathrm{~m} / \mathrm{s}\left(E_{\mathrm{kin}}=6.4 \mathrm{~cm}^{-1}\right)$.
\end{abstract}

DOI: 10.1103/PhysRevA.78.030702

PACS number(s): $34.50 .-\mathrm{s}, 37.10 . \mathrm{Mn}, 29.20 . \mathrm{dk}, 29.27 . \mathrm{Bd}$

Collisions between molecules are highly sensitive to temperature, especially in the low-temperature regime where the kinetic energy of the molecules is comparable to the rotational energy level splittings in the collision complex. At these energies, translational energy can be transferred into internal energy, effectively binding the molecules transiently together. These long-lived excitations of the collision complex appear as sharp resonances in graphs of the cross section as a function of the collision energy. As the position and width of the resonances provide an extremely sensitive probe of the interaction potential energy surfaces [1-3], measurements of these resonances are an important goal in experimental cold molecule research. Recently, Gilijamse et al. have presented a crossed-beam scattering experiment between Stark-decelerated $\mathrm{OH}$ molecules and Xe atoms [4]. In this experiment, the total center-of-mass collision energy was varied from 50 to $400 \mathrm{~cm}^{-1}$ with an energy resolution of $13 \mathrm{~cm}^{-1}$. Both the lowest attainable energy and the energy resolution were limited by the properties of the xenon beam. This can be improved upon by using two decelerated beams; however, obtaining the required densities in the scattering region poses a major challenge. A way to increase the number of collisions that will take place is to load the decelerated beams into a molecular synchrotron. As opposed to the crossed-beam geometry, in which the particles encounter each other only once, in a synchrotron the particles meet many times. Losses due to elastic and inelastic collisions from the counterpropagating packets will result in a decrease of the number of stored molecules that scales with the number of round trips. This provides a sensitive probe of the total collisional cross section. We have recently demonstrated a molecular synchrotron capable of confining one (or two) packet(s) of ammonia molecules flying at one specific velocity in a tight bunch [5]. In order to use the synchrotron as a low-energy collider, it is imperative that the velocity of the stored molecules can be tuned over a wide range. Due to the bunching elements in a synchrotron, instabilities arise at certain velocities of the stored molecules. These instabilities are well known in charged particle accelerator physics; in fact, great efforts are exerted in the design of a synchrotron to minimize these so-called stopbands or motional resonances [6]. Recently, similar instabilities were observed in a storage ring for paramagnetic atoms [7]. In this Rapid Communication, we present an experimental study of mo- tional resonances occurring in a molecular synchrotron, and show that by appropriately changing the voltages applied to the ring electrodes we can store molecules over a wide velocity range.

Our experimental setup is schematically shown in Fig. 1. A pulsed beam of deuterated ammonia is formed by expanding a mixture of about $5 \% \mathrm{ND}_{3}$ in xenon into vacuum through a cooled solenoid valve. In the molecular beam, only the lowest rotational levels in the electronic and vibrational ground state of $\mathrm{ND}_{3}$ are occupied. The experiments are performed with molecules that reside in the low-field-seeking $|J, K M\rangle=|1,-1\rangle$ level, which constitutes about $20 \%$ of all the $\mathrm{ND}_{3}$ molecules in the beam. A hexapole focuses the molecules into the 63-stage Stark decelerator, which decelerates them to velocities between 60 and $90 \mathrm{~m} / \mathrm{s}$. Two hexapoles and a buncher are used to focus the decelerated packet into the synchrotron. The combination of the decelerator and the focusing elements allows for complete control over the spatial and velocity distributions of the beam; further details of the injection beamline can be found elsewhere [8]. The synchrotron consists of two hexapole focusers bent into a semicircle separated by a $2 \mathrm{~mm}$ gap. The ring radius $R_{\text {ring }}$ is $125 \mathrm{~mm}$. The electrodes have a $4 \mathrm{~mm}$ diameter, and are positioned symmetrically on the outside of a circle with a radius $R_{\text {hex }}$ of $4 \mathrm{~mm}$. The $\mathrm{ND}_{3}$ molecules are detected at the injection point in the synchrotron using $(2+1)$ resonanceenhanced multiphoton ionization (REMPI) with pulsed laser light around $317 \mathrm{~nm}$.



FIG. 1. Experimental setup. A pulsed beam of $\mathrm{ND}_{3}$ molecules is decelerated, bunched, and focused into a molecular synchrotron. The two half rings have a radius of $125 \mathrm{~mm}$; they are separated by a gap of $2 \mathrm{~mm}$. Molecules in the detection region are ionized using pulsed laser light around $317 \mathrm{~nm}$, extracted perpendicularly to the plane of the ring, and counted by an ion detector. 
As the molecules circle around the ring, appropriate voltages are applied to confine and bunch the molecules. For transverse confinement, positive and negative voltages are applied to adjacent electrodes. This voltage configuration results in an electric field that is zero at the center of the hexapole and increases quadratically away from the center. Molecules with a positive Stark shift will experience a force toward the center of the hexapole. In a curved hexapole the molecules will, in addition, experience a centrifugal force pushing them outward. At a certain radius the electrostatic force and the centrifugal force will cancel. This radius, which is velocity dependent, is referred to as the equilibrium radius $r_{\text {equi }}^{\prime}$. In a ring without gaps—a storage ring $[9,10]-\mathrm{a}$ molecule will remain at this equilibrium radius as it revolves around the ring and will form a closed orbit. Molecules flying with the same forward velocity but with a different radial position or with a nonzero radial velocity will oscillate around this closed orbit. The motion in this transverse well is called the betatron oscillation in accelerator physics [6].

For longitudinal confinement, a voltage difference is applied between the two half rings as the molecules pass through the gaps. The voltages are switched in synchronization with the position and velocity of one hypothetical molecule, appropriately referred to as the synchronous molecule, such that the molecules in front of this molecule are decelerated and the molecules lagging behind this molecule are accelerated. As a result, the molecules within a small position and velocity interval will experience a force toward the synchronous molecule and will oscillate around it; the molecules are trapped in a traveling potential well that revolves around the ring. The motion in this longitudinal well is called the synchrotron oscillation in accelerator physics [6].

Introduction of the gaps is necessary for bunching the molecules; however, it affects the transverse motion of the molecules. In the gap, the confinement force temporarily vanishes. The disturbance on the molecules' trajectory as the result of one gap is small, and is, in itself, not necessarily a problem. However, if the disturbance accumulates the consequences will become disastrous. Calculated trajectories are shown in Fig. 2 for a molecule with a longitudinal velocity $v_{\phi}$ of $76 \mathrm{~m} / \mathrm{s}$ [Figs. 2(a) and 2(b)] and $82 \mathrm{~m} / \mathrm{s}$ [Figs. 2(c) and 2(d)]. The voltage difference between adjacent electrodes, $V$, is taken to be $7.5 \mathrm{kV}$. In Fig. 2(a), the radial position of a molecule flying with a longitudinal velocity of $76 \mathrm{~m} / \mathrm{s}$ is plotted as a function of time. At this velocity, the equilibrium orbit is displaced by a distance $r_{\text {equi }}^{\prime}=1.9 \mathrm{~mm}$ from the geometrical center of the hexapole. The molecule whose trajectory is plotted in Fig. 2(a) began at an initial radial position $r^{\prime}$ different from $r_{\text {equi }}^{\prime}$; hence it oscillates around the equilibrium radius. At a voltage difference of $7.5 \mathrm{kV}$ the betatron frequency is around $850 \mathrm{~Hz}$. In phase space, shown in Fig. 2(b), this oscillation results in an ellipse. The molecule moves in a clockwise direction around the position and velocity of the closed orbit (located at the intersection of the gray dashed axes). When the molecule is in a gap, it experiences no confinement force and thus it moves along a straight horizontal line in phase space; the trajectory in a gap is denoted by an arrow. The length and direction of the displacement is determined by the velocity of the molecule as it enters the gap. The molecule plotted in
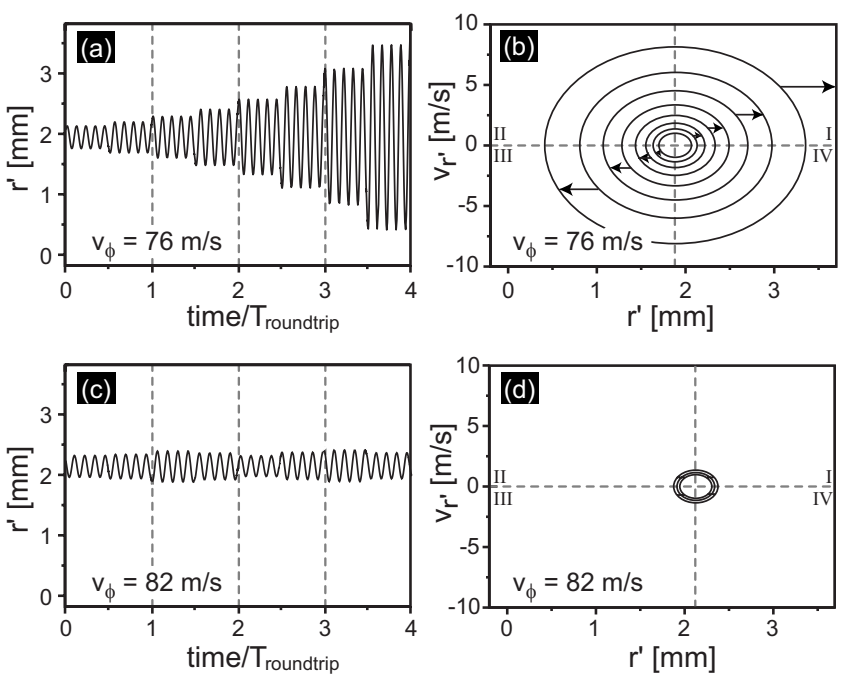

FIG. 2. Radial trajectories of two molecules moving at 76 (a) and $82 \mathrm{~m} / \mathrm{s}$ (c) completing four round trips in a synchrotron. The same trajectories are plotted in phase space in (b) and (d), respectively. At a voltage difference of $7.5 \mathrm{kV}$, a molecule moving at a velocity of $76 \mathrm{~m} / \mathrm{s}$ makes 4.5 oscillations as it completes one-half of a round trip. In this case, the amplitude of the betatron oscillation grows each time the molecule passes the gap until it is lost from the ring. A molecule moving at a velocity of $82 \mathrm{~m} / \mathrm{s}$ makes 4.1 oscillations as it completes one-half of a round trip. In this case, the amplitude of the molecule's betatron oscillation is modulated, but the overall result on the trajectory averages out.

Figs. 2(a) and 2(b) has a positive velocity and a positive displacement from the equilibrium orbit as it passes the first gap, i.e., the molecule is in quadrant I. This means it will move away from the equilibrium orbit. After the gap, the molecule is captured again and now moves along a larger ellipse; the molecule now oscillates with a larger amplitude. At a forward velocity of $76 \mathrm{~m} / \mathrm{s}$ the molecule oscillates 4.5 times around the equilibrium orbit every half round trip. This means that, as the molecule passes the next gap, it has a negative velocity and a negative displacement from the equilibrium orbit, i.e., the molecule is in quadrant III. The molecule again moves away from the equilibrium orbit and the amplitude of its betatron oscillation further increases. As the molecule continues to revolve, it is always located in either quadrant I or III as it enters a gap, and the molecule's oscillation amplitude continues to increase until it is ejected from the ring.

In Fig. 2(c) and 2(d) similar graphs are shown for a molecule with a longitudinal velocity of $82 \mathrm{~m} / \mathrm{s}$. Again the molecule oscillates around the equilibrium orbit, which at this velocity is displaced by $2.1 \mathrm{~mm}$ from the geometrical center of the hexapole. For $82 \mathrm{~m} / \mathrm{s}$, the molecule oscillates 4.1 times around the equilibrium orbit every half round trip. As the molecule passes the first two gaps, it is located in quadrants I and III, and therefore its amplitude increases. However, as it passes the third gap, it is located in quadrant IV. This means that the molecule now moves toward the equilibrium orbit, and the amplitude of its betatron oscillation actually decreases. In this way, as the molecule continues to revolve its betatron oscillation amplitude varies, but the 
overall effect of the gaps averages out, leaving the molecule stably confined.

From this discussion, we conclude that the number of betatron oscillations a molecule makes between the gaps is decisive for the stability of its trajectory. In accelerator physics this number is called the betatron tune $\nu$ [6]. Motional resonances occur when the betatron tune is equal to a halfinteger value (including integer values). In order to stably confine molecules, the synchrotron needs to be operated at a tune that is sufficiently far away from these values. Note that, although close to a half-integer value the trajectory is mathematically stable, the amplitude varies widely. If the amplitude at some point is larger than the aperture of the ring, the molecule will be lost from the ring. The width of the resonance, therefore, scales with the length of the gap and is inversely proportional to the aperture of the ring.

It is instructive to look at how the betatron tune scales with respect to the molecule's longitudinal velocity and the applied voltage difference between the ring electrodes. The betatron tune is given by $\nu=\omega / n \Omega$, where $\omega / 2 \pi$ is the betatron frequency, $\Omega / 2 \pi$ is the cyclotron frequency, and $n$ is the number of repeating units in the ring (in our case $n=2$ ). Both the betatron and cyclotron frequencies have a complicated dependence on $v_{\phi}$ and $V$; however, a simple formula can be derived using the following approximations: (i) the radius of the ring is much larger than the aperture, i.e., $R_{\text {ring }} / R_{\text {hex }} \gg 1$, (ii) the electric field is perfectly harmonic, and (iii) the Stark shift is positive and linear, $W_{\text {Stark }}=\mu_{\text {eff }}|E|$, where $|E|$ is the absolute value of the electric field and $\mu_{\text {eff }}$ is the effective dipole moment of the molecule. Under these approximations the tune can be written as

$$
\nu=\frac{R_{\text {ring }}}{n v_{\phi}} \sqrt{\frac{\mu_{\text {eff }}}{m} \frac{3 V}{R_{\text {hex }}^{3}}},
$$

where $m$ is the mass of the molecule. From this equation, we see that in order to keep the tune at a constant value, we need to adjust $V$ proportionately to $v_{\phi}^{2}$. Note that, when the tune is held constant, the equilibrium radius, approximately given by $[10]$

$$
r_{\text {equi }}^{\prime}=\frac{v_{\phi}^{2}}{R_{\text {ring }} \frac{\mu_{\text {eff }}}{m} \frac{3 V}{R_{\text {hex }}^{3}}},
$$

remains constant as well. This is important as it implies that the optimal position to inject and detect molecules does not change as a function of velocity.

As explained in Ref. [5], we increase the voltages applied to the electrodes by $30 \%$ immediately before and after the molecules pass through the gap. In this way the lack of confinement in the gaps is partly compensated and the strength of the stopbands is reduced [10]. This so-called corrector configuration also compensates for misalignments in the ring.

Figure 3(a) shows measurements of the density of injected ammonia molecules as a function of their longitudinal velocity. For each data point, a new time sequence is loaded with the optimum timings for switching the electric fields throughout the injection beamline. Each measurement is av-
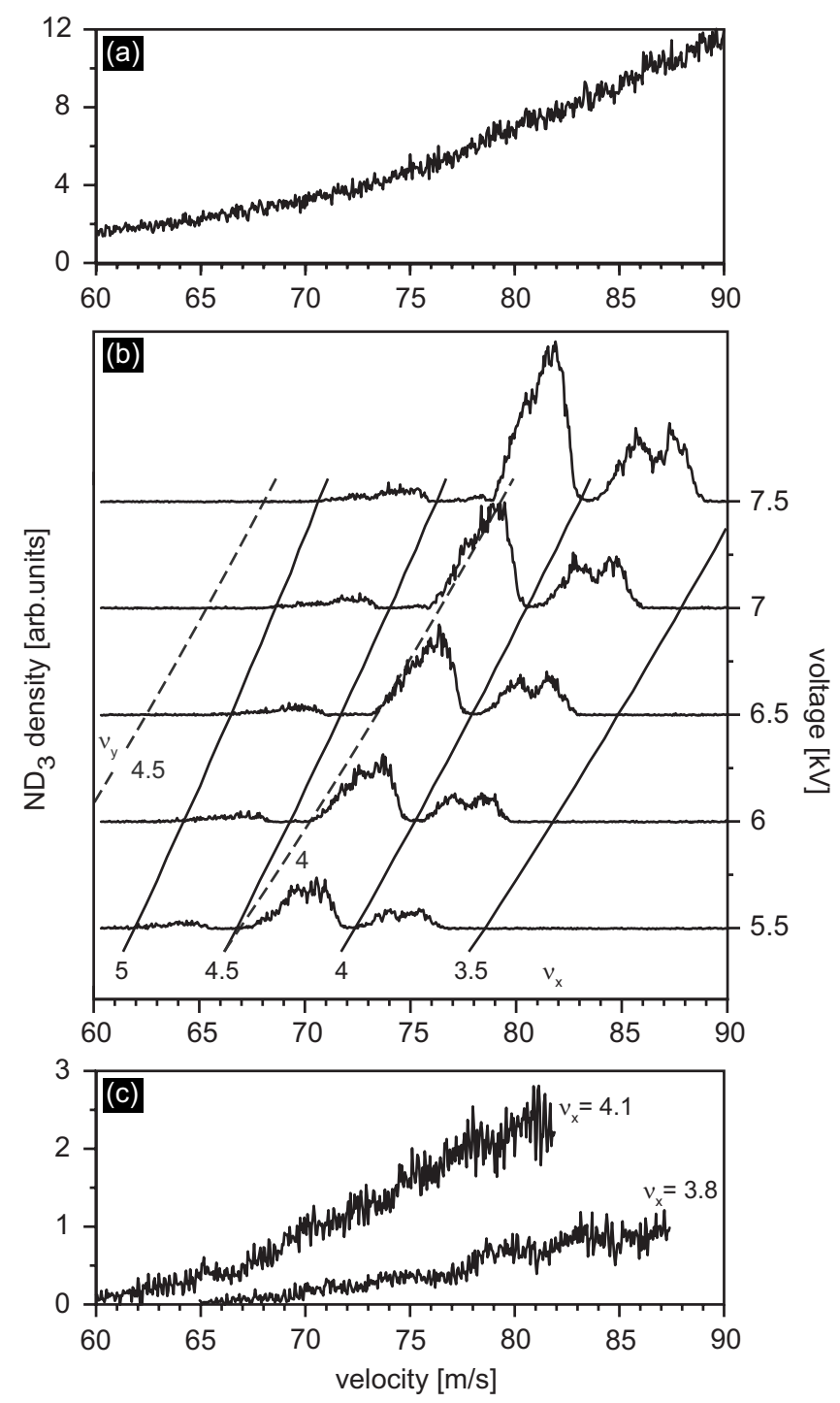

FIG. 3. Density of $\mathrm{ND}_{3}$ molecules at injection (a) and upon completion of the 20th round trip (b) as a function of the molecules' longitudinal velocity measured at five different voltages. The solid and dashed lines indicate when the horizontal and vertical betatron tunes have a half-integer value, respectively. In (c), the density of $\mathrm{ND}_{3}$ molecules upon completion of the 20th round trip is shown as a function of the molecules' longitudinal velocity, but now with the voltages changed in synch with the velocity such as to keep the horizontal betatron tune constant at either 4.1 or 3.8 as indicated.

eraged over 96 shots. The longitudinal velocity spread of the packet is on the order of $1 \mathrm{~m} / \mathrm{s}$. The number of molecules exiting the decelerator does not depend on the final velocity; however, due to the finite aperture of the hexapoles used in the beamline, the number of molecules loaded into the ring decreases at lower velocities [8].

In Fig. 3(b), the density of ammonia molecules is again shown as a function of velocity, but now after the molecules have completed 20 round trips in the ring. The measurements are taken at five different ring voltages as indicated on the right-hand side of the figure. Similar measurements taken after the 40th round trip (not shown) look identical after the additional losses due to collisions with background gas are 
taken into account. We first turn our attention to the upper measurement, taken at $V=7.5 \mathrm{kV}$. Molecules are injected into the ring at approximately $2 \mathrm{~mm}$ from the geometrical center of the hexapole, which is the equilibrium radius for around $80 \mathrm{~m} / \mathrm{s}$ at this voltage difference. In the absence of stopbands, we expect to observe a broad distribution of molecules centered around $80 \mathrm{~m} / \mathrm{s}$. It is seen, however, that only molecules with velocities centered around 74, 81, and $86 \mathrm{~m} / \mathrm{s}$ are stored, while molecules with velocities around 76 and $83 \mathrm{~m} / \mathrm{s}$ are lost from the ring. Using the electric field from a finite-element program [11] and the known Stark shift of $\mathrm{ND}_{3}$ (taking a dipole moment of $1.48 \mathrm{D}$ [12]), we have calculated the betatron tune as a function of the applied voltage and the longitudinal velocity of the molecules. The solid and dashed lines in Fig. 3(b) indicate when the horizontal and vertical betatron tunes have a half-integer value, respectively. Note that within the approximations made to derive Eq. (1) the horizontal and vertical tunes are identical. Apart from the (strong) half-integer resonances due to the bunching elements, additional (weaker) resonances may arise due to imperfections and misalignments of the ring. Every round trip, as the molecule passes a particular defect in the ring, its trajectory will be slightly disturbed. These disturbances will accumulate when the molecules make a half-integer number of oscillations per round trip. These resonances are found by setting $n=1$ in Eq. (1). The dip observed around $86 \mathrm{~m} / \mathrm{s}$ results from a resonance occurring when the molecules make 7.5 oscillations per round trip. As the voltages are lowered the observed profile of the velocity scans is seen to shift to lower velocities. Although the exact velocities of the calcu- lated stopbands is not always in perfect agreement with observed dips in the velocity scans, it is evident that the stopbands indeed explain all the observed structure.

Figure 3(c) again shows the density of ammonia molecules after completing 20 round trips, but now the voltages are varied for each velocity such that the horizontal betatron tune $\nu_{x}$ is always equal to either 4.1 or 3.8 as indicated in the figure. In the measurement where $\nu_{x}$ is kept at 4.1 (3.8), the voltage difference is lowered from $7.7 \mathrm{kV}$ at 82 $(87.5) \mathrm{m} / \mathrm{s}$ to $3.8 \mathrm{kV}$ at $60(65) \mathrm{m} / \mathrm{s}$. As can be seen, the density of the stored molecules now resembles that of the injected beam shown in Fig. 3(a). For the measurement where $\nu_{x}$ is kept at 4.1 , the signal at the 20th round trip at $82 \mathrm{~m} / \mathrm{s}$ is almost 4 times lower, and at $60 \mathrm{~m} / \mathrm{s}$ about 20 times lower, than that of the injected beam. The decrease at lower velocities is due to the shallower longitudinal trapping potential at lower voltages.

In this Rapid Communication, we demonstrate that molecules can be stored with different velocities in a molecular synchrotron. We observe motional resonances similar to those observed in charged particle accelerators. We show that these resonances can be understood and predicted from a simple model, and that they can be avoided by appropriately changing the voltages. This is crucial for the ability to perform collision studies in a molecular synchrotron.

We thank D. Carty for useful discussions. H.L.B. acknowledges financial support from the Netherlands Organisation for Scientific Research (NWO) via a VENI grant.
[1] N. Balakrishnan, A. Dalgarno, and R. C. Forrey, J. Chem. Phys. 113, 621 (2000).

[2] E. Bodo and F. A. Gianturco, Int. Rev. Phys. Chem. 25, 313 (2006).

[3] J. M. Hutson and P. Soldan, Int. Rev. Phys. Chem. 26, 1 (2007).

[4] J. J. Gilijamse, S. Hoekstra, S. Y. T. van de Meerakker, G. C. Groenenboom, and G. Meijer, Science 313, 1617 (2006).

[5] C. E. Heiner, D. Carty, G. Meijer, and H. L. Bethlem, Nat. Phys. 3, 115 (2007).

[6] S. Y. Lee, Accelerator Physics, 2nd ed. (World Scientific, Singapore, 2004).
[7] K. W. Murch, K. L. Moore, S. Gupta, and D. M. StamperKurn, Phys. Rev. Lett. 96, 013202 (2006).

[8] C. E. Heiner, H. L. Bethlem, and G. Meijer, Phys. Chem. Chem. Phys. 8, 2666 (2006).

[9] F. M. H. Crompvoets, H. L. Bethlem, R. T. Jongma, and G. Meijer, Nature (London) 411, 174 (2001).

[10] F. M. H. Crompvoets, H. L. Bethlem, and G. Meijer, Adv. At., Mol., Opt. Phys. 52, 209 (2005).

[11] D. A. Dahl, Computer code SIMION 3D Version 6.0 (Idaho National Engineering Laboratory, Idaho Falls, 1995).

[12] R. L. Bhattacharjee, L. H. Johnston, G. R. Sudhakaran, and J. C. Sarker, J. Mol. Spectrosc. 138, 38 (1989). 